\title{
Simple analysis of water-filled hollow-core silica photonic bandgap fiber
}

\author{
Hirokazu Kubota $^{1 \mathrm{a})}$, Satoki Kawanishi ${ }^{1,3}$, and Masaya Notomi ${ }^{2}$ \\ ${ }^{1}$ NTT Network Innovation Laboratories, Yokosuka, Kanagawa 239-0847, Japan \\ 2 NTT Basic Research Laboratories, Atsugi, Kanagawa 243-0124, Japan \\ ${ }^{3}$ Current address: Tamagawa University Research Institute, Machida, Tokyo \\ 194-8610, Japan
}

a)kubota.hirokaszu@lab.ntt.co.jp

\begin{abstract}
We performed a simple numerical investigation of a silica hollow core photonic bandgap fiber whose core area was filled with water. We propose cladding parameters for the PBF designed to obtain a photonic bandgap of broader than $200 \mathrm{~nm}$ at a center wavelength of $500 \mathrm{~nm}$.
\end{abstract}

Keywords: hollow-core PBF, capillary

Classification: Optical fiber

\section{References}

[1] J. D. Joannopoulos, R. D. Meade, and J. N. Winn, Photonic Crystals: Molding the Flow of Light, Princeton U. Press, West Sussex, 1995.

[2] P. Roberts, F. Couny, H. Sabert, B. Mangan, D. Williams, L. Farr, M. Mason, A. Tomlinson, T. Birks, J. Knight, and P. St. J. Russell, "Ultimate low loss of hollow-core photonic crystal fibres," Opt. Express, vol. 13, no. 1, pp. 236-244, Jan. 2005.

[3] T. Larsen, A. Bjarklev, D. Hermann, and J. Broeng, "Optical devices based on liquid crystal photonic bandgap fibres," Opt. Express, vol. 11, no. 20, pp. 2589-2596, Oct. 2003.

[4] F. M. Cox, A. Argyros, and M. C. J. Large, "Liquid-filled hollow core microstructured polymer optical fiber," Opt. Express, vol. 14, no. 9, pp. 4135-4140, May 2006.

[5] A. Wang, A. K. George, and J. C. Knight, "Three-level neodymium laser incorporating photonic bandgap fiber," Opt. Lett., vol. 31, no. 10, pp. 1388-1390, May 2006.

[6] R. Goto, et al., "Cladding-pumped Yb-doped solid photonic bandgap fiber for ASE suppression in shorter wavelength region," Proc. OFC/NFOEC 2008, San Diego, USA, OTuJ5, Feb. 2008.

[7] A. Argyros, T. Birks, S. Leon-Saval, C. M. Cordeiro, F. Luan, and P. St. J. Russell, "Photonic bandgap with an index step of one percent," Opt. Express, vol. 13, no. 1, pp. 309-314, Jan. 2005.

[8] S. Kawanishi, T. Komukai, M. Ohmori, and H. Sakaki, "Photoluminescence of semiconductor nanocrystal quantum dots at $1550 \mathrm{~nm}$ wavelength in the core of photonic bandgap fiber," Proc. CLEO/QELS 200\%, Baltimore, USA, CTuII4, May 2007. 
[9] S. Kawanishi, M. Ohmori, M. Tanaka, and H. Sakaki, "Observation of photoluminescence of semiconductor nanocrystal quantum dots in the core of photonic bandgap fiber," Tech. Digest of 19th LEOS annual meeting, Montreal, Canada, ThN 4, Oct. 2006.

[10] M. Ohmori, S. Kawanishi, M. Tanaka, and H. Sakaki, "Photoluminescence spectroscopy of semiconductor colloidal quantum dots in the photonic bandgap fiber," Tech. Digest of IEEE LEOS Winter Topicals Conf. 2008, Sorrento, Italy, paper MD2.2, Jan. 2008.

[11] [Online] http://ab-initio.mit.edu/wiki/index.php/MIT_Photonic_Bands

[12] T. A. Bricks, D. M. Atkin, G. Wylangowski, P. S. J. Russel, and P. J. Roberts, "2D Photonic band gap structures in fiber form," Photonic Band Gap Materials, ed. C. M. Soukoulis, pp. 437-444, Kluwer Academic Publishers, Dordrecht, 1996.

[13] F. Luan, A. K. George, T. D. Hedley, G. J. Pearce, D. M. Bird, J. C. Knight, and P. St. J. Russell, "All-solid photonic bandgap fiber," Opt. Lett., vol. 29, no. 20, pp. 2369-2371, Oct. 2004.

\section{Introduction}

Conventional optical fiber confines light in its core region by employing a total inner reflection (TIR) mechanism, which means that the refractive index of the core must be higher than that of the cladding. Unlike this fiber, photonic bandgap fiber (PBF) confines light by means of the photonic bandgap effect. In this case the refractive index of the core can be lower than that of the cladding, and the core can even be hollow.

The photonic bandgap is a feature of periodic dielectric structures through which light in a certain frequency range cannot propagate [1]. A onedimensional type is known as Bragg reflection, and a two- or three- dimensional type is called photonic crystal.

In $\mathrm{PBF}$, the core is surrounded by two-dimensional photonic crystal cladding. For certain wavelengths, light with a wave vector component along the fiber that matches the propagation constant in the core material, is prevented from propagating through the cladding by the photonic bandgap effect. Therefore light at these wavelengths cannot escape from the core.

A typical PBF is a silica hollow core photonic crystal fiber, which has a triangular arrangement of holes in its cladding. The core is a hole larger than those in the cladding, which is formed by removing typically 7 or 19 unit cells from the cladding. One aim with PBF was to realize optical fiber with an ultimately low loss and PBF loss has now reached $1.2 \mathrm{~dB} / \mathrm{km}[2]$. At the same time, it is expected that high performance optical devices will be achieved by filling the holes in the PBF with various materials. Liquid crystal photonic bandgap fiber is being investigated to realize tunable optical devices $[3,4]$ whose core and cladding holes are filled with liquid crystal.

There is another type of PBF, which has solid cladding. Solid silica based PBF is being studied with a view to realizing high power fiber lasers by employing the filtering effect of the $\mathrm{PBF}[5,6]$. The cladding of this type of PBF consists of an alignment of high index rods. The band structure of this 
$\mathrm{PBF}$ is rather simple and suitable for use in gaining a physical understanding of the guiding mechanism. A bandgap fiber with a refractive index contrast of only a few percent has been achieved using silica core fiber [7].

Hollow core PBF can be used as a capillary and carry gas or liquid in its core area. A semiconductor nano-particle quantum dot is an attractive material. The operating wavelength of a quantum dot is controlled by its size. A PBF core can be filled with nano-particles in the form of a water solution $[8,9,10]$.

Water and alcohols are common solvents and the refractive indexes of water and methanol are about 1.33 and 1.36, respectively. As they are lower than that of $\mathrm{SiO}_{2}$ glass, the neff value of the propagating mode is not always higher than that of fundamental space filling mode (FSM). In such a case, the photonic bandgap is essential for confining light stably in the fiber.

In this paper, we study PBF with a water-filled core and found the cladding structure to be suitable for guiding visible light.

\section{Models}

We studied silica-based hollow core PBF with three types of cladding. The first one is conventional PBF, which has air holes aligned in the cladding. The second one has holes filled with high refractive index material. It resembles an all-solid bandgap fiber but its core remains hollow. The last structure is kagome lattice. We assume a simple model where all the elements are aligned perfectly. We also assume that the refractive indices of the materials are constant over the investigated wavelength region.

The core region is formed by removing 19 rods. The diameter of the core area was as large as $4.3 \Lambda$, and filled with water. It has a large core volume compared with single-mode fiber. We ignore the slight differences between the refractive index of the core material and the propagating mode for simplicity.

The band structure is calculated by the plane wave expansion method using MPB [11]. We used a fine mesh of about 100 lines / unit cell because we need to evaluate the bandgap at a $\lambda / \Lambda$ of around 0.1 , and we used about 10 lines per wavelength for accurate modeling.

Figure 1 (a) shows a typical drawing [12] of the band structure of kagome structure PBF, where wall thickness $w$ was $0.05 \Lambda$. We can determine the bandgap frequencies at $n=1$, and also at $n=1.33$ by drawing an appropriate line as shown in the figure. However, it is difficult to read the bandgap frequencies for various $n$ values. Figure 1 (b) shows the normalized wavelength versus the refractive index. When the core area of a hollow core PBF is filled with various materials while the cladding is unchanged, the band structure remains the same. Therefore, it is easy to determine the bandgap wavelength when changing core materials using this figure. 

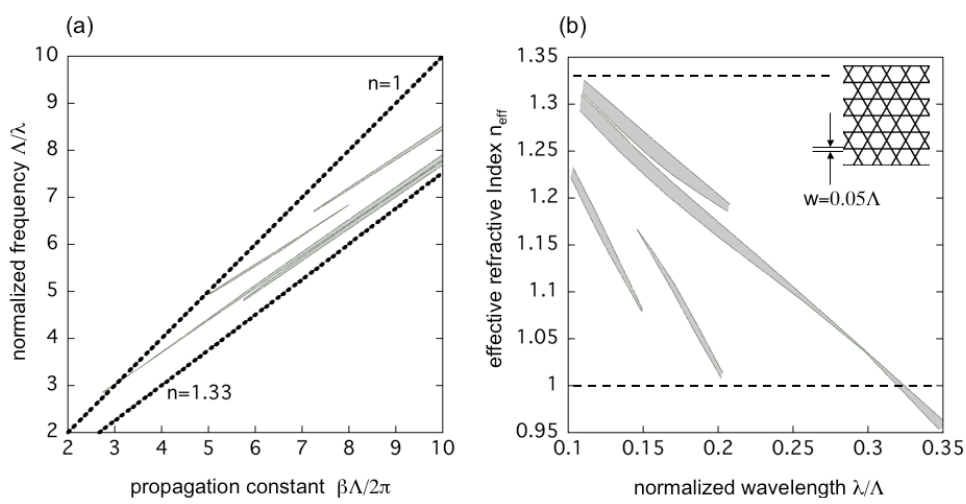

Fig. 1. Band structure of kagome PBF shown with (a) frequency vs. propagation constant (b) $\mathrm{n}_{\mathrm{eff}}$ vs. wavelength. Wall thickness $w$ was $0.05 \Lambda$. Broken lines indicating $\mathrm{n}=1$ and $\mathrm{n}=1.33$ are also shown.

\section{Numerical results}

First, we investigate PBF with high refractive index fillings, as all-solid PBFs [5, 6, 7] and liquid crystal PBFs have already been reported. [3, 4] Liquid crystals or flint glass [13] are candidates as high index materials for filling the cladding holes. Figure 2 (a) shows the band structure in effective refractive index $\left(\mathrm{n}_{\text {eff }}\right)$ versus the normalized wavelength, $\lambda / \Lambda$, where the filling material had refractive index of 1.8. The inset shows a cross-section of the structure. White indicates silica and black indicates high refractive index material. The hole diameter $d / \Lambda$ was 0.5 . We focus on an $n_{\text {eff }}$ of 1.33 , which is indicated by the dotted line in the figure.

Figure $2(\mathrm{~b})$ shows the bandgap at $\mathrm{n}_{\mathrm{eff}}=1.33$ for various $d / \Lambda$ values, where the cladding holes are filled using materials with refractive index of 1.8. There is a fundamental bandgap at $\lambda / \Lambda$ grater than 1 . That is, the hole pitch should smaller than the wavelength. If the diameter of the filled region is half of the hole pitch, it is difficult to fill such small holes with liquid crystal or flint glass.

The hole pitch and thus the hole diameter can be enlarged by using a high order bandgap. This figure indicates the existence of a bandgap at around $\lambda / \Lambda=0.6$ and 0.8 , but the bandgap opening is narrow compared with the fundamental bandgap. We performed calculations for various refractive indexes of filled material and we found that this structure needs filling material with an refractive index of at least 1.6 to achieve bandgap opening with a water core. Using a high refractive index material as a filling provides a wide bandgap opening but this results in an FSM with a high $\mathrm{n}_{\text {eff }}$. It is difficult to realize a bandgap with a low propagation constant in this type of PBF, because the FSM cannot be lower than that of $\mathrm{SiO}_{2}$. Reducing the refractive index of the base material, such as polymer, may be a possible solution, but we do not deal with such non-glass based PBFs here.

Next, we investigated the band structure of conventional air-silica PBF as shown in Fig. 3 (a), where d is $0.95 \Lambda$. The insets shows the cladding structure and mode profile of the fundamental core mode mode at a wavelength 

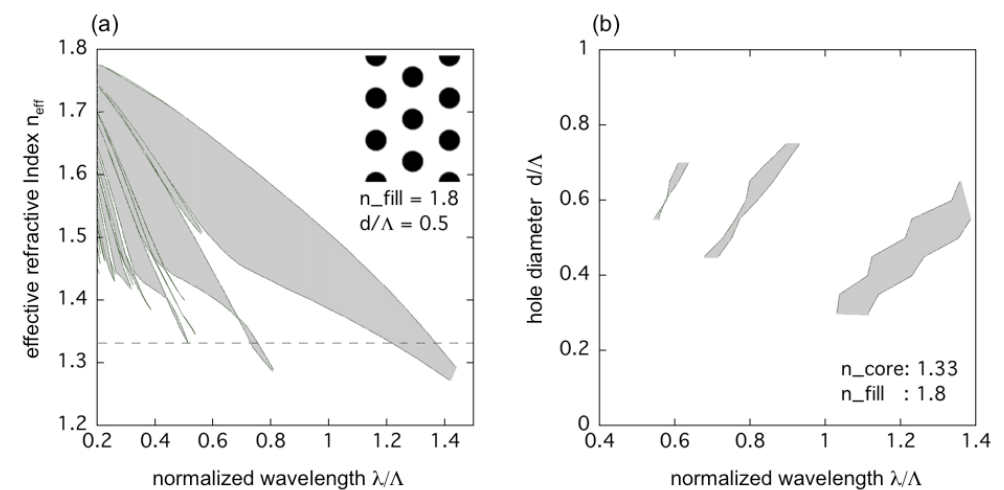

Fig. 2. (a) Band structure and bandgap wavelength of PBF where $d / \Lambda=0.5$ and the refractive index of the filling was 1.8. The inset shows a cross-section of the analyzed structure. White indicates silica and black indicates a high refractive index filling. (b) Bandgap wavelength of the PBF for various $d / \Lambda$.

of $550 \mathrm{~nm}$. The air fraction of the cladding was $82 \%$. The modal index of the fundamental mode with a water-filled core, was calculated to be about 1.3295. The refractive indices of the propagating modes are almost the same as that of the core material in this large core fibers. The figure indicates the presence of a high order bandgap at shorter wavelengths than the fundamental bandgap, but here we focus solely on the fundamental bandgap. The bandgap wavelength of the air guiding mode $\left(n_{\text {eff }}=1\right)$ is around $0.6 \Lambda$ and it shifts towards a shorter wavelength as the core index increases. At $\mathrm{n}_{\text {eff }}=$ 1.33 , there is a bandgap between $0.150 \Lambda$ and $0.233 \Lambda$. When $\Lambda=2.6 \mu \mathrm{m}$ for example, the wavelength is 380 to $606 \mathrm{~nm}$.

(a)

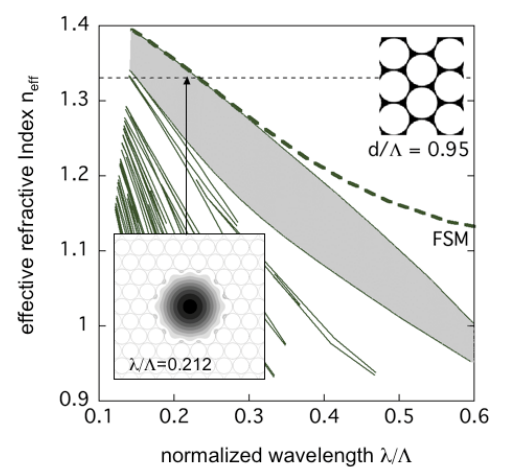

(b)

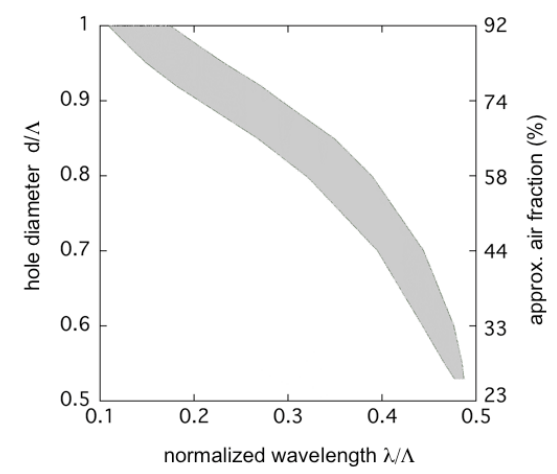

Fig. 3. (a) Band structure of conventional PBF where $d / \Lambda$ is 0.95 . The insets show the cladding structure and fundamental core mode when core is filled with water. FSM of the cladding structure is shown by a thick broken line. (b) Fundamental bandgap wavelength of the PBF for various $d / \Lambda$. 
The FSM of the cladding structure is also shown by a thick broken line. The figure indicates that the $\mathrm{n}_{\text {eff }}$ of the FSM is always higher than that of the bandgap region. That is, the confinement mechanism is purely the bandgap when the frequency is within the bandgap region.

This bandgap region shifts towards a shorter wavelength as $d / \Lambda$ increases as shown in Fig. 3 (b). When the center wavelength of the bandgap is fixed at $500 \mathrm{~nm}$ by changing $\Lambda$, the wavelength range of the bandgap broadens as $d / \Lambda$ increases. A bandwidth broader than $200 \mathrm{~nm}$ at a center frequency of $500 \mathrm{~nm}$ can be achieved when $d / \Lambda>0.93$.

We also investigated a kagome lattice as shown in Fig. 1 (b). We investigate various wall thicknesses $w$ from $0.01 \Lambda$ to $0.1 \Lambda$. The thin wall will cause a bandgap opening at a low $\mathrm{n}_{\text {eff }}$ due to the low glass concentration. The thick wall can increase the $n_{\text {eff }}$ value but has a small bandgap opening compared with that in Fig. 3 (a). We conclude that kagome structure is unsuitable for the present purpose.

\section{Conclusion}

We investigated silica hollow core PBF with a water-filled core area. We show that a bandwidth of more than $200 \mathrm{~nm}$ at a center wavelength of $500 \mathrm{~nm}$ is possible in a simple PBF structure when $d / \Lambda>0.93$. The center wavelength can be tuned by varying the hole pitch and the bandwidth can be changed by selecting the $d / \Lambda$ value.

\section{Acknowledgments}

The authors would like to thank S. Matsuoka for his encouragement. 